\title{
Elimination of the data-dependent defects by doubling the frequency of driving signals for cholesteric LCDs
}

\author{
A. Rybalochka \\ V. Lashkaryov Institute of Semiconductor Physics, NAS of Ukraine \\ 45, prospect Nauky, 03028 Kyiv, Ukraine \\ Phone: +38 (044) 525-97-86; e-mail: andriy.rybalochka@gmail.com
}

\begin{abstract}
Using the standard waveform composition method for $2+2$ dynamic drive schemes results in the dependence of image contrast on image data-content. In this paper, an implementation of this standard method with doubling the frequency of driving signals is proposed. It allows to equalize the effective selection time for all display's pixels independently of preceding and following non-selected pixel voltage waveforms. Thus, data-pattern-dependent defects at the passive-matrix addressing of cholesteric liquid crystal displays are fully eliminated.
\end{abstract}

Keywords: bistable display, cholesteric liquid crystal, dynamic drive scheme.

Manuscript received 18.12.09; accepted for publication 25.03.10; published online 30.04.10.

\section{Introduction}

Cholesteric liquid crystal displays (ChLCDs) are widely investigated in recent years due to paper-like look and ultra-low power consumption [1-3]. These displays don't need any power and backlight for storage and representtation of displayed images due to inherent to them longterm bistability and good sunlight readability. Bistable reflective ChLCDs are usually used in low power applications where frequent update of information is not required, such as e-book, e-paper, price tickets, etc.

There are two stable states of a cholesteric liquid crystal (ChLC): the planar state $(P)$ that reflects light at a preselected wavelength determined by the pitch of cholesteric composition, and the focal-conic state $(F C)$ that is weakly scattered or nearly optical transparent. Refreshing information for a ChLCD is switching ChLC in display's pixels between these two stable states. It can be achieved by ac voltage pulses [4]. Under low-voltage pulses, cholesteric material is driven from the $P$ - to the $F C$-state and remains after the pulse. Under a voltage higher than the critical value of the cholesteric-nematic transition, ChLC is driven to the homeotropic state $(H)$. From the $H$-state, it can relax to both $P$ - and $F C$ - stable states. If the high voltage is switched to a middle voltage level, ChLC transforms to the stable $F C$-state. Fast switching-off the voltage allows ChLC to transform to the final stable $P$-state. The transition from the $H$-state to $P$-state takes place in two steps: first, the homeptropic state - the transient planar state $\left(P^{*}\right)$ transition and, second, the transient planar state - the stable planar state transition. The transition from the $H$-state to $P^{*}$-state ( $H-P^{*}$ transition) is of the order of $1 \mathrm{~ms}$, while the other relaxation transitions are of the order of $100 \mathrm{~ms}$. The stable $F C$-state can be obtained from the $P^{*}$-state by applying the middle-voltage pulses to ChLC, too. It is important that for the fixed pulse duration there is a voltage range in which the $H$-state is held and the $P$-, $F C$ - and $P^{*}$-states are addressed to the final $F C$-state by the same voltage pulse. This fact demonstrates hysteresis properties of ChLCDs $[5,6]$.

Various dynamic drive schemes (DDSs) with addressing speed about $1 \mathrm{~ms}$ per line have been developed as based on the features described above for the field induced state transitions of ChLCs and their hysteresis properties. First DDS requires very complicated electronic driving circuitry, when all column and row drivers must output bipolar and multilevel voltages [7]. Moreover, during the image writing, because of a pipeline algorithm used with this drive scheme, there is an undesirable black bar shifting over the frame. A unipolar waveform composition method has been adopted to implement above mentioned DDS [8]. However, since the amplitude of voltages required in different addressing stages are distinct, both row and column drivers are required to generate multi-level unipolar voltages. The simplest driving voltage waveforms are realized in 2+2 DDSs [9-11]. In these 
drive schemes, only two voltage levels are used in driving voltage waveforms that applied to rows and columns of a display. In this case, both row and column drivers are required to generate only unipolar signals with two levels: $U_{\text {Peak }}$ and zero. The composite across pixel voltage waveforms have three voltage levels: $U_{P e a k}$, $-U_{\text {Peak }}$ and zero. These drive schemes do not produce undesirable black shifting bars during addressing. The entire frame goes black during writing, and than image appears on full display's area at once.

When addressing the ChLCD by $2+2$ DDS, the dependence of image contrast on image data-content is observed. The presence of these data-pattern-dependent defects lies in dependence of the effective selection time on preceding and following non-selected voltage waveforms. Thus, the final reflective state of ChLC in pixels can be changed in undesirable way.

In this paper, we propose an improvement of the "framing voltage pulse" method [12] and new "doubling the frequency" method for an elimination of the datapattern-dependent defects at passive-matrix addressing the ChLCD. Elimination of defects is achieved by equalization of the effective selection time for all display's pixels for any image data-content.

\section{Data-pattern-dependent defects}

The pipeline algorithm for $2+2$ DDSs is shown in Fig. 1 . There are four addressing stages in these DDSs: the preparation stage, pre-selection and post-selection parts of the holding stage, the selection stage and the evolution stage. In the first preparation stage $\left(U_{P r}, T_{P r}\right)$, the value of the preparation voltage is sufficient to transform $\mathrm{ChLC}$ into the $H$-state in all pixels irrespective of its initial state. In the pre-selection part of the holding stage $\left(U_{H d}, T_{H d}{ }^{\text {pre-S }}\right), \mathrm{ChLC}$ is kept in the $H$-state. The determination of the final state of ChLC occurs during the selection stage $\left(U_{S}^{\text {on }}\right.$ or $\left.U_{S}^{\text {off }}=0, T_{S}\right)$. The selection time must be not less than the $H-P^{*}$ transition time. Thus, in selected pixels $\left(U_{S}^{o n}\right) \mathrm{ChLC}$ is kept in the $H$ state and in non-selected pixels $\left(U_{S}^{o f f}\right)$ ChLC transforms into the $P^{*}$-state. In the post-selection part of the holding stage and in the evolution stage $\left(U_{E v}=U_{H d}, T_{E v}\right)$, ChLC remains in the $H$-state, if it was kept in this state during the selection stage. When ChLC is transformed into the $P^{*}$-state during the selection stage, it evolves to the final $F C$-state. When the driving sequences are finished in non-selected pixels, the stable $F C$-state is formed, and in selected pixels ChLC transforms from the $H$-state to the stable $P$-state. The summarized duration of driving sequences is equal to the frame time: $T_{\text {Frame }}=T_{P r}+N T_{S}$ $+T_{E v}$, where $N$ is the number of rows in the display. When $N$ is large (about 1000 rows), the average time to address one row is $T_{\text {Frame }} / N \approx T_{s}$.

There are no problems to obtain necessary effective voltages and stage durations for all the pixels during the preparation and evolution stages, because of the same effective voltages are applied to all the display's pixels simultaneously in these stages. The main problem is to

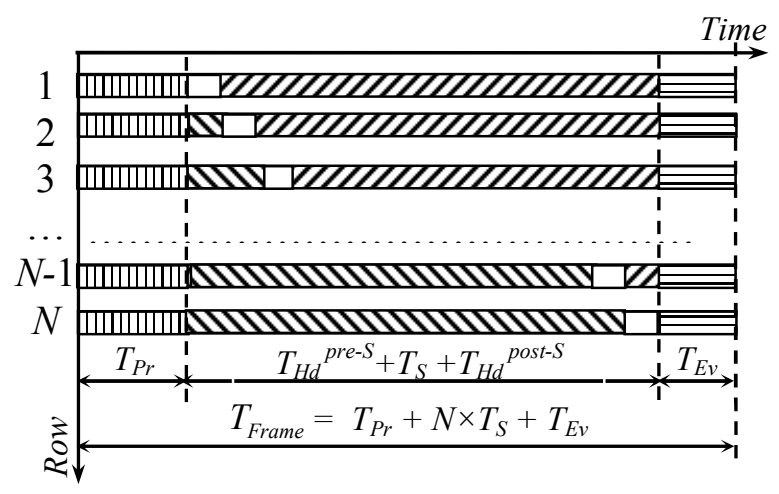

Fig. 1. The pipeline algorithm for $2+2$ DDS.

compose driving signals for the selection and holding stages, because when pixels from one row are in the selection stage pixels from all other rows are in the holding stage. Moreover, when a row is in the selection stage, two different effective voltages $\left(U_{S}{ }^{o n}\right.$ and $\left.U_{S}{ }^{\text {off }}\right)$ may be applied to pixels from this row simultaneously. In Fig. 2 the standard concept of the waveform composition method for 2+2 DDS for the selection and holding stages is presented. Waveforms for selected row $\left(R_{S}\right)$, non-selected rows $\left(R_{N / S}\right)$, selected columns $\left(C_{S}\right)$ and non-selected columns $\left(C_{N / S}\right)$ give four voltage waveforms for pixels: $P_{H d 1}$ and $P_{H d 2}$ - two possible waveforms for pixels in the holding stage, $P_{S}^{\text {on }}$ and $P_{S}^{o f f}$ - selected and non-selected waveforms for pixels in the selection stage, correspondingly. These waveforms have duration equal to the selection time $\left(T_{S}\right)$ and effective voltage levels: $U_{P e a k} / \sqrt{ } 2$ for $P_{S}^{o n}, P_{H d 1}, P_{H d 2}$; and zero for $P_{S}^{\text {off }}$. In this DDS, before and after the selection stage one from the holding waveforms $\left(P_{H d 1}\right.$ or $\left.P_{H d 2}\right)$ is presented. In Table 1 , the effective selection time $\left(T_{S}^{e f f}\right)$ for four possible combinations of $P_{H d 1}, P_{H d 2}$ and $P_{S}^{\text {off }}$ is presented. Since the selection time in DDSs is very short (about $1 \mathrm{~ms}$ ), a small change in it has big impact on the optical properties. Thus, variation of the effective selection time causes unplanned variations in the brightness of the dark $(F C)$ state, and as the result leads to uneven contrast ratio.

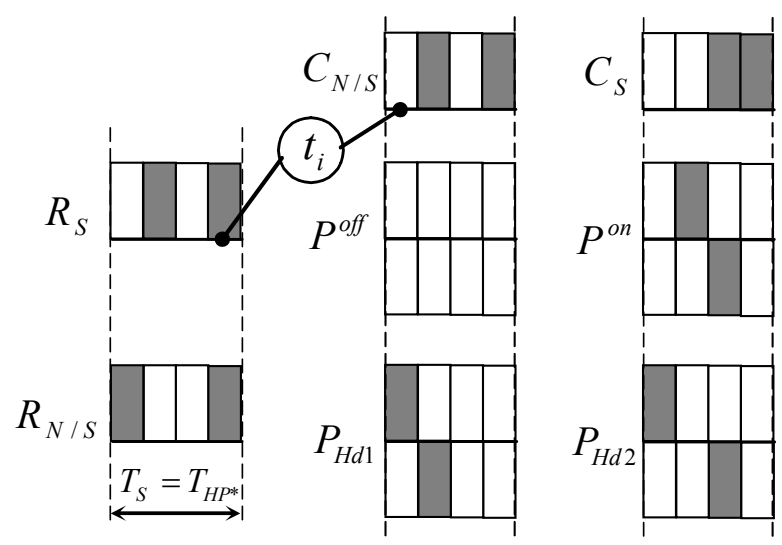

Fig. 2. The standard concept for $2+2$ DDS. 
Table 1. Possible effective selection times for the standard waveform composition method.

\begin{tabular}{|c|c|c|}
\hline № & Combinations & $T_{S}^{\text {eff }} / T_{S}$ \\
\hline 1 & $P_{H d 1}+P^{\text {off }}+P_{H d 1}$ & 1.5 \\
\hline 2 & $P_{H d 1}+P^{\text {off }}+P_{H d 2}$ & 1.5 \\
\hline 3 & $P_{H d 2}+P^{o f f}+P_{H d 1}$ & 1.25 \\
\hline 4 & $P_{H d 2}+P^{o f f}+P_{H d 2}$ & 1.25 \\
\hline
\end{tabular}

\section{Improved "framing voltage pulse" method}

To reduce effective selection time variation, the "framing voltage pulse", method was proposed [12]. In this method, frame waveforms are inserted between selected rows. It means that after a row is selected, no row is selected and fixed data is presented on the rows and columns. Fig. 3 shows two variants of frame waveforms $\left(P_{F 1}\right.$ or $\left.P_{F 2}\right)$ with corresponding rows and columns voltage pulses. Now effective selection time is constant: for the combination " $P_{F 1}+P^{o f f}+P_{F 1}$ " it is $T_{S}$, and for the combination " $P_{F 2}+$ $P^{\text {off }}+P_{F 2}$ " it is $1.5 \times T_{S}$ (see Fig. 4). As a result, the data dependence of image contrast is eliminated. However, application of this method increases the frame time practically by two times, and the average addressing speed is about $2 T_{S}$ per row. However, it can be reduced in case of using the $P_{F 2}$ waveform. The duration of each subsignal and segment for presented in Figs 2 to 4 waveforms is determined as $t_{i}=T_{H-P^{*}} / 4$. Thus, the selection time duration is $T_{S}=4 \times t_{i}=T_{H-P^{*}}$, and the condition for dynamic addressing the ChLCD is proved. For combination " $P_{F 2}+P^{o f f}+P_{F 2}$ " at $t_{i}=T_{H-P^{*}} / 6$, the effective selection time is equal to $T_{H-P^{*}}$, and the selection time is $T_{S}=(2 / 3) \times T_{H-P^{*}}$. Thus, the total frame time can be decreased by one thirdth, and the averaged addressing speed is about $(4 / 3) \times T_{S}$ per row. As a result, the improved "framing voltage pulse" method allows to realize no-datadependent-defects addressing with the frame time increasing only by $33 \%$ in comparison with the standard waveform composition method.
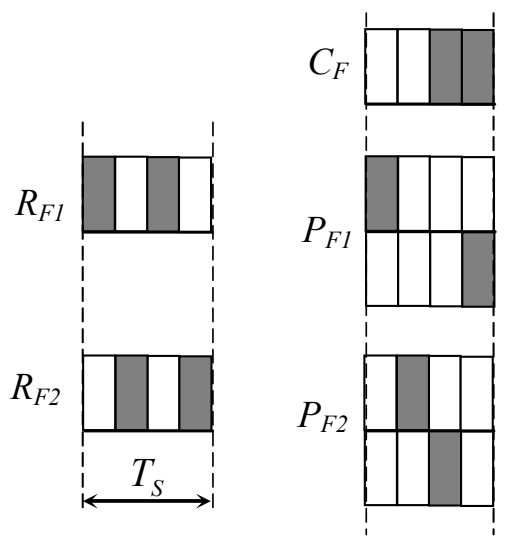

Fig. 3. Two variants of frame waveforms.

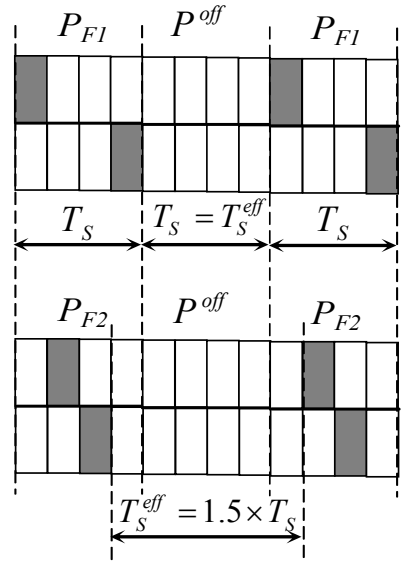

Fig. 4. The concept of the "framing voltage pulse" method.

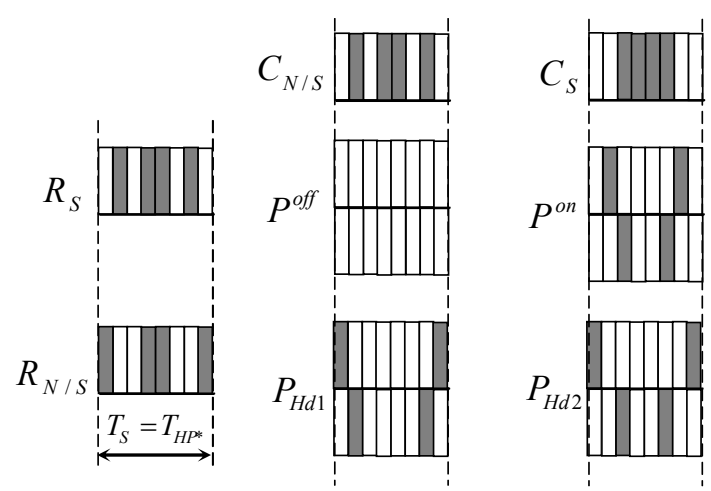

Fig. 5. The concept of the "doubling the frequency" method.

\section{4. "Doubling the frequency" method}

There is a solution of the data-pattern-dependent defect problem without any increasing the frame time. It needs only two times increasing the frequency of signals in driving pulses. The concept of this "double the frequency" method is shown in Fig. 5. Driving signals in this method are symmetrically repeated voltage pulses from Fig. 2 with doubled frequency. Now for any combination from Table 1, there is only one value of the effective selection time $-T_{S}$, which is independent of the non-selected pixel voltages proceeding and following a selected row. And moreover, the average time to address one row is the same as for the standard waveform composition method and is equal $T_{S}$.

Table 2. The comparison of methods for elimination of the data-pattern-dependent defects.

\begin{tabular}{|c|c|c|}
\hline $\begin{array}{c}\text { Waveform composition } \\
\text { method }\end{array}$ & $\begin{array}{c}\text { Relative } \\
\text { addressing } \\
\text { speed }\end{array}$ & $\begin{array}{c}\text { Data- } \\
\text { dependent- } \\
\text { defects }\end{array}$ \\
\hline "Standard" [9-11] & 1 & Yes \\
\hline "Framing voltage pulse" [12] & 2 & No \\
\hline $\begin{array}{c}\text { "Framing voltage pulse" } \\
\text { (improved) (this paper) }\end{array}$ & 1.33 & No \\
\hline $\begin{array}{c}\text { "Doubling the frequency" } \\
\text { (this paper) }\end{array}$ & 1 & No \\
\hline
\end{tabular}


Summarized results of previously known and presented in this paper methods for elimination of the data-pattern-dependent defects are presented in Table 2. The compared parameters are: the relative addressing speed - the ratio of the average addressing speed of certain method to the average addressing speed for the standard waveform composition method, and "presenceabsence" of the data-pattern-dependent defects. We can see from this table that the methods proposed in this paper allow to eliminate the data-pattern-dependent defects.

The improved "framing voltage pulse" method has the addressing speed by one thirdth less than the frame time for the standard waveform composition method. And the new "doubling the frequency" method allows to eliminate data-pattern-dependent defects without increasing the frame time in general.

\section{Conclusion}

The problem of data-pattern-dependent defects resulted from the standard concept of two-level dynamic drive scheme for cholesteric LCD has been analyzed. Proposed early the "framing voltage pulse" method solves this problem with two times increase in the frame time. In this paper, an improvement of this method has been proposed. Equalization of the effective selection time to the homeotropic - transient planar transition time allows to eliminate data-dependent-defects with increasing the frame time only by $33 \%$. Moreover, the new "doubling the frequency" method has been proposed, too. It demonstrates the possibility of solving the discussed problem without any increasing the frame time as compared with the standard waveform composition method.

\section{References}

1. X.Y. Huang, A. Khan, Reflective cholesteric display technology and its application // Proc. ASID 2002, p. 211-213 (2002).
2. I. Dozov, Bistable liquid crystal technologies // SID 2003 Digest, p. $946-949$ (2003).

3. J.W. Doane, D. Davis, A. Khan, E. Montbach, T. Schneider, I. Shiyanovskaya, Cholesteric reflective displays: thin and flexible // Proc. IDRC 2006, p. 9-12 (2006).

4. X.Y. Huang, D.K. Yang, J.W. Doane, Transient dielectric study of bistable reflective cholesteric displays and design of rapid drive scheme // Appl. Phys. Lett. 67(9), p. 1211-1213 (1995).

5. M. Kawachi, O. Kogure, Hysteresis behavior of texture in field induced nematic-cholesteric relaxation // Jpn. J. Appl. Phys. 16(9), p. 673-1678 (1977).

6. A. Rybalochka, V. Sorokin, M. Minyaylo, Control of width of hysteresis characteristics for ChLC // Proc. 13 $13^{\text {th }}$ Intern. Symp. "Advanced Display Technologies”, p. 61-65 (2004).

7. X.Y. Huang, D.K. Yang, P. Bos and J.W. Doane, Dynamic drive for bistable reflective cholesteric displays: a rapid addressing scheme // SID 1995 Digest, p. 347-350 (1995).

8. X.Y. Huang, N. Miller, J.W. Doane, Unipolar implementation for the dynamic drive scheme of bistable reflective cholesteric displays // SID 1997 Digest, p. 899-902 (1997).

9. A. Kozachenko, P. Oleksenko, V. Sorokin, V. Nazarenko, Histeresis as a key factor for the fast control of reflectivity in cholesteric LCDs // Proc. IDRC 1997, p. 148-151 (1997).

10. V. Sorokin, Simple driving methods for cholesteric reflective LCDs // Proc. IDRC 1998, p. 749-752 (1998).

11. A. Rybalochka, V. Sorokin, S. Valyukh, A. Sorokin, V. Nazarenko, Simple drive scheme for bistable cholesteric LCDs // SID 2001 Digest, p. 882-885 (2001).

12. X.D. Mi, D.M. Johnson, S.W. Stephenson, Dynamic driving schemes for cholesteric LCDs to eliminate data-dependent defects // SID 2007 Digest, p. 57-60 (2007). 